\title{
Hypoxia-mediated activation of autophagic flux inhibits apoptosis of keratinocytes via blocking tumor necrosis factor-related apoptosis-inducing ligand
}

\author{
SUNG-WOOK KIM and SANG-YOUEL PARK \\ Department of Biochemistry, Biosafety Research Institute, College of Veterinary Medicine, \\ Chonbuk National University, Jeonju, Jeonbuk 561-756, Republic of Korea \\ Received October 13, 2014; Accepted June 30, 2015
}

DOI: $10.3892 / \mathrm{mmr} .2015 .4592$

\begin{abstract}
Tumor necrosis factor-related apoptosis-inducing ligand (TRAIL) is toxic against transformed tumor cells. Cornification is the terminal differentiation of keratinocytes and a specific form of programmed cell death caused by TRAIL that occurs in keratinocytes. Apoptosis can also be triggered when TRAIL induces expression of keratinocyte differentiation markers. The present study reported that hypoxia inhibits TRAIL-induced apoptosis due to autophagic flux. It is well known that hypoxia activates autophagy in keratinocytes and reduces p62 protein levels. The present study demonstrated that hypoxia inhibited TRAIL-mediated apoptosis and induced autophagic flux in HaCaT cells. In addition, autophagic flux-inactivating reagents, including 3-methyladenine and chloroquine, increased the TRAIL sensitivity of HaCaT cells exposed to hypoxia. In conclusion, these results indicated that inactivating autophagy increased TRAIL sensitivity in hypoxic HaCaT cells. Autophagy inhibitors may be beneficial in therapies using TRAIL against skin cancers.
\end{abstract}

\section{Introduction}

Tumor necrosis factor (TNF)-related apoptosis-inducing ligand (TRAIL) is a member of the TNF superfamily. Four types of death receptors specific for TRAIL have been found: Death receptor 4 (DR4), DR5 and decoy receptors DCR1 and DCR2. Death receptors mediate TRAIL-induced cell death, whereas the decoy receptors inhibit death signaling (1). The increased sensitivity of transformed cells to TRAIL-induced cell death compared with that of normal cells suggests the potential of

Correspondence to: Professor Sang-Youel Park, Department of Biochemistry, Biosafety Research Institute, College of Veterinary Medicine, Chonbuk National University, 664-14 Duk-Jin Dong 1-Ga, Jeonju, Jeonbuk 561-756, Republic of Korea

E-mail: sypark@chonbuk.ac.kr

Key words: hypoxia, autophagy, tumor necrosis factor-related apoptosis-inducing ligand, keratinocyte
TRAIL to treat various cancer types. Furthermore, TRAIL induces cell death in normal human epidermal keratinocytes (2). In addition, human TRAIL can induce tissue injury (as cell death, inflammation) in human endothelial cells (3).

Hypoxia is a common environmental stressor. Hypoxia-inducible factor 1 (HIF-1) is a transcriptional factor composed of $\alpha$ - and $\beta$-sub-units that mediates changes in gene expression at low oxygen concentrations (4). Targets of HIF-1 $\alpha$ include cytokines and growth factors, as well as molecules involved in angiogenesis, glucose uptake and metabolism, and cell survival $(5,6)$. In addition, HIF-1 $\alpha$ is essential for adaptation of cells to environmental stress and has an important role in skin development and wound healing $(7,8)$. HIF-1 $\alpha$ is strongly expressed in skin epithelium. Human and mouse skin is hypoxic, with normal oxygen levels of 1.5-5.0\% $(9,10)$. However, unlike numerous internal tissue types, human epidermis gains much of its oxygen supply from the atmosphere and experiences higher oxygen levels than those of internal tissues (11). Of note, hypoxia activates autophagic flux and induces clearance of the p62 protein, suggesting a role for p62 in the regulation of hypoxic HaCaT-cell survival responses (12).

Autophagy is a strictly controlled program in which parts of the cytoplasm are sequestered in double membrane autophagosome vesicles, which fuse with lysosomes to form autolysosomes (13). Lysosomes degrade protein aggregates, aged proteins and cytoplasmic organelles $(14,15)$. Oxidative stress induces the accumulation of high-weight protein aggregates containing the autophagy marker protein p62 in autophagy-deficient keratinocytes (16). In addition, blocking autophagic flux significantly increased inflammatory cytokine levels and p62 protein expression in primary human keratinocytes (17). These studies suggested that blocking autophagic flux (p62 protein accumulation) is involved in increased inflammation and induced cell death.

In the present study, human HaCaT keratinocytes were stimulated with hypoxia, and the TRAIL-induced expression of autophagy markers, including LC3 and p62, as well as apoptosis were assessed. Furthermore, the effects of the autophagic flux inhibitors 3-methyladenine (3-MA) and chloroquine (CQ) on autophagy marker expression and $\mathrm{HaCaT}$ cell viability were assessed. The present study indicated that autophagy inhibitors may increase the anti-cancer efficiency of TRAIL. 


\section{Materials and methods}

Cell culture. HaCaT cells were obtained from the American Type Culture Collection (Manassas, VA, USA) and maintained in Dulbecco's modified Eagle's medium (Gibco; Thermo Fisher Scientific, Inc., Waltham, MA, USA) supplemented with $10 \%$ (v/v) fetal bovine serum (FBS; Gibco; Thermo Fisher Scientific, Inc.) and antibiotics $(100 \mu \mathrm{g} / \mathrm{ml}$ gentamycin and $100 \mu \mathrm{g} / \mathrm{ml}$ penicillin-streptomycin; Gibco; Thermo Fisher Scientific, Inc.). A hypoxia chamber was used to create the low-oxygen environment composed of $1 \% \mathrm{O}_{2}, 5 \% \mathrm{CO}_{2}$ and $94 \% \mathrm{~N}_{2}$.

Protein isolation and western blot analysis. Total protein was isolated from harvested cells (using lysis buffer comprising phenylmethanesulfonyl fluoride, $\mathrm{Na}_{3} \mathrm{VO}_{4}$ and complement $\mathrm{C}$ ), suspended in phosphate-buffered saline (PBS). The pellets were re-suspended and sonicated in buffer (Sigma-Aldrich, St. Louis, MO, USA) containing $20 \mathrm{mM}$ Tris, $\mathrm{pH} 7.5,1 \%$ Triton X-100, 1 mM EDTA, 1 mM ethylene glycol tetraacetic acid (EGTA) as well as protease and phosphatase inhibitors (Sigma-Aldrich). The lysates were subjected to western blot analysis. Total protein from $\mathrm{HaCaT}$ cells was isolated by homogenization in cold radioimmunoprecipitation assay buffer (Sigma-Aldrich) containing $50 \mathrm{mM}$ Tris, $\mathrm{pH} 7.5$, $150 \mathrm{mM}$ sodium chloride, $1 \% \mathrm{NP}-40,0.5 \%$ sodium deoxycholate, $0.1 \%$ SDS, $0.1 \mathrm{mM}$ EDTA and $0.1 \mathrm{mM}$ EGTA, as well as the appropriate protease and phosphatase inhibitors. The lysates were centrifuged and the supernatants were subjected to western blot analysis.

Protein concentrations were estimated using a protein assay (Thermo Fisher Scientific, Inc.) according to the manufacturer's instructions. Nitrocellulose (NC, Merck Millipore, Milford, MA, USA) membranes were incubated with 5\% nonfat milk to block non-specific binding. The membranes were subsequently exposed to antibodies that recognized polyclonal LC3 (cat. no. 4108; Cell Signaling Technology, Inc., Danvers, MA, USA); monoclonal P62 (cat. no. MABC32; Merck Millipore) and monoclonal HIF-1 $\alpha$ (cat. no. sc-53546; Santa Cruz Biotechnology, Inc., Dallas, TX, USA). The primary antibodies were incubated at a dilution of 1:1,000 in $5 \%$ bovine serum albumin or $5 \%$ non-fat milk for $18 \mathrm{~h}$ at $4{ }^{\circ} \mathrm{C}$. The membranes were exposed to polyclonal goat anti-rabbit immunoglobulin (Ig)G (cat. no. ADI-SAB-300-j; Enzo Life Sciences, Inc., Farmingdale, NY, USA), polyclonal goat anti-mouse IgG (cat. no. ADI-SAB-100-j; EnzoLife Sciences, Inc.) or anti-goat secondary antibodies conjugated with horseradish peroxidase (dilution, 1:10,000 for all) in Tris-buffered saline containing Tween-20 (TBS-T) and 5\% nonfat milk for $1 \mathrm{~h}$ at room temperature. Electrophoresis was performed using an electrophoresis chamber (Bio-Rad Laboratories, Inc., Hercules, CA, USA). A Westsave Gold enhanced chemiluminescence kit (AbFrontier; Young In Frontier Co., Ltd., Seoul, South Korea) was used, and the signals were detected using a chemiluminescence detection system (Fusion Fx7 version 15.18; Vilber Lourmat, Eberhardzell, Germany) and exposed to X-ray film.

Crystal violet assay. Cell viability was determined by crystal violet staining (Sigma-Aldrich), as described previously (18). Briefly, HaCaT cells were pre-incubated under hypoxic conditions $\left(1 \% \mathrm{O}_{2}\right.$ for $\left.24 \mathrm{~h}\right)$ and exposed to $100-400 \mathrm{ng} / \mathrm{ml}$ TRAIL (AbFrontier; Young In Frontier Co., Ltd.) for $6 \mathrm{~h}$. The cells were pre-treated with autophagy inhibitors [200 $\mu \mathrm{M}$ 3-MA (Sigma-Aldrich) or $50 \mu \mathrm{M} \mathrm{CQ}$ (Sigma-Aldrich)] for $3 \mathrm{~h}$ and exposed to $200 \mathrm{ng} / \mathrm{ml}$ TRAIL for $6 \mathrm{~h}$ under hypoxic $\left(1 \% \mathrm{O}_{2}\right.$ for $\left.24 \mathrm{~h}\right)$ or normoxic conditions. Cell viability was calculated based on the relative dye intensity compared with that of the controls.

Lactate dehydrogenase ( $L D H)$ assay. Cytotoxicity was assessed by the LDH assay using the supernatant and a LDH Cytotoxicity Detection kit (Takara Bio Inc., Tokyo, Japan) according to the manufacturer's instructions. LDH activity was determined by measuring the absorbance at a wavelength of $490 \mathrm{~nm}$ using a SpectraMax M Series spectrophotometer.

Statistical analysis. Values are expressed as the mean \pm standard error. Results of different treatments and time courses were analyzed using one-way analysis of variance. Comparisons between two groups were analyzed by two-tailed Student's $t$-test, analysis of variance and Duncan's multiple range test using the SAS statistical package 9.1 (SAS Institute, Cary, NC, USA)

\section{Results}

Hypoxia inhibits TRAIL-induced apoptosis of HaCaT cells. $\mathrm{HaCaT}$ cells were cultured under hypoxic or normoxic conditions and treated with TRAIL $(100-400 \mathrm{ng} / \mathrm{ml})$. Morphological examination of the cell population by microscopy (Eclipse TS100; Nikon Corporation, Tokyo, Japan) indicated decreased TRAIL-induced apoptosis under hypoxia as compared with normoxia (Fig. 1A). The influence of hypoxia on TRAIL-induced apoptosis in $\mathrm{HaCaT}$ cells was then quantified by crystal violet staining (Fig. 1B). HaCaT cells were responsive to TRAIL treatment (10-60\% reduction in cell viability) under normoxic conditions, whereas under hypoxic conditions, TRAIL only had a minor effect (5\% reduction in cell viability) on cell viability (Fig. 1C), indicating that hypoxia prevented TRAIL-induced apoptosis. Consistent with these results, the LDH assay also showed that hypoxia prevented TRAIL-induced apoptosis (Fig. 1D). These results confirmed that hypoxia prevented TRAIL-mediated apoptosis.

HIF-1 $\alpha$ induces the expression of autophagy markers in hypoxic HaCaT cells. HIF-1 $\alpha$ is a transcription factor and a major regulator of cell adaptation to low oxygen levels. The present study evaluated HIF- $1 \alpha$ protein levels in $\mathrm{HaCaT}$ cells under hypoxic and normoxic conditions by western blot analysis. Cells were incubated under hypoxic conditions (1\% $\mathrm{O}_{2} ; 0,6,12$ or $24 \mathrm{~h}$ ), and a western blot analysis was performed to determine HIF-1 $\alpha$ protein levels (Fig. 2A). Cells displayed increased HIF-1 $\alpha$ protein levels under the hypoxic conditions but not under normoxia. It is known that mild hypoxia activates autophagy in keratinocytes and reduces p62 protein levels (12). Autophagy controls cell survival, growth and cellular homeostasis as well as cellular defense. Thus, the present study assessed the autophagy marker, LC3 and the protein, P62 that is cleared by autophagic flux, by western 

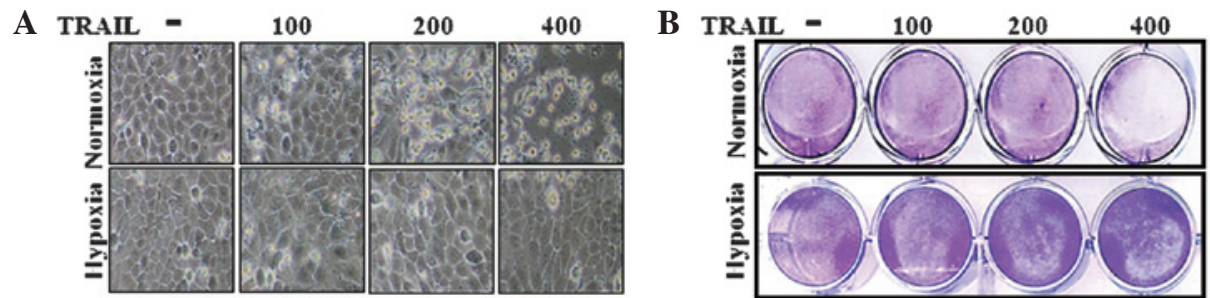

C

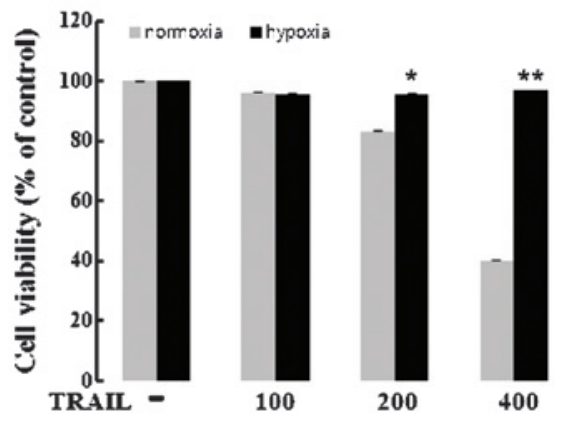

D

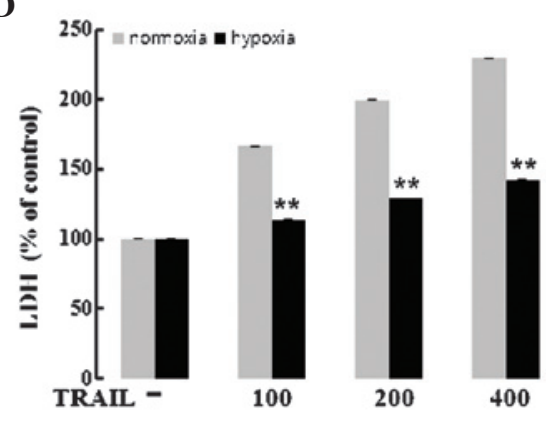

Figure 1. Hypoxia inhibits TRAIL-induced apoptosis in HaCaT cells. (A) Cell morphology was observed by light microscopy (magnification, x200). HaCaT cells were exposed to $100-400 \mathrm{ng} / \mathrm{ml}$ TRAIL for $6 \mathrm{~h}$ under normoxia or hypoxia $\left(1 \% \mathrm{O}_{2}\right.$ for $\left.24 \mathrm{~h}\right)$. (B and C) The viability of treated cells was assessed by crystal violet staining. The viability of control cells was $100 \%$. HaCaT cells were pre-incubated under hypoxic conditions ( $1 \%$ O for $24 \mathrm{~h})$ and then exposed to $200 \mathrm{ng} / \mathrm{ml}$ TRAIL for $6 \mathrm{~h}$. (D) The cells were treated with 100-400 ng/ml TRAIL for $6 \mathrm{~h}$ under normoxia, and LDH release into the cell culture medium was measured after exposure to hypoxia for $24 \mathrm{~h}$. Values are expressed as the mean \pm standard error $(\mathrm{n}=2)$. ${ }^{* *} \mathrm{P}<0.01 \mathrm{vs}$. normoxia. LDH, lactate dehydrogenase; TRAIL, tumor necrosis factor-related apoptosis-inducing ligand.
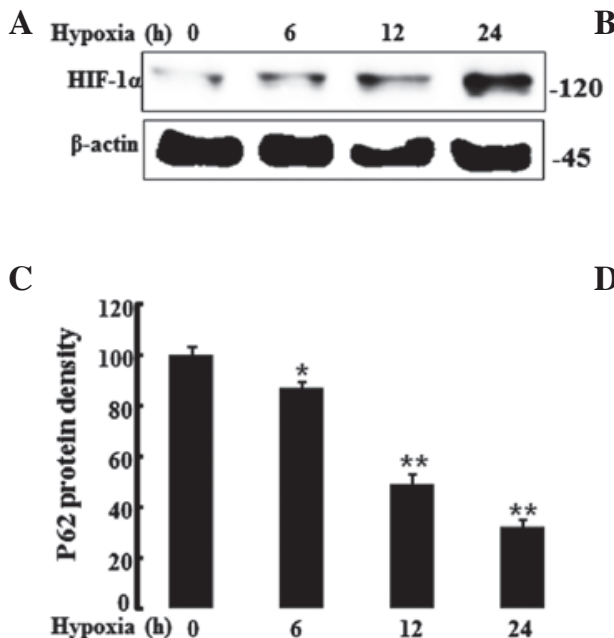

\section{B}
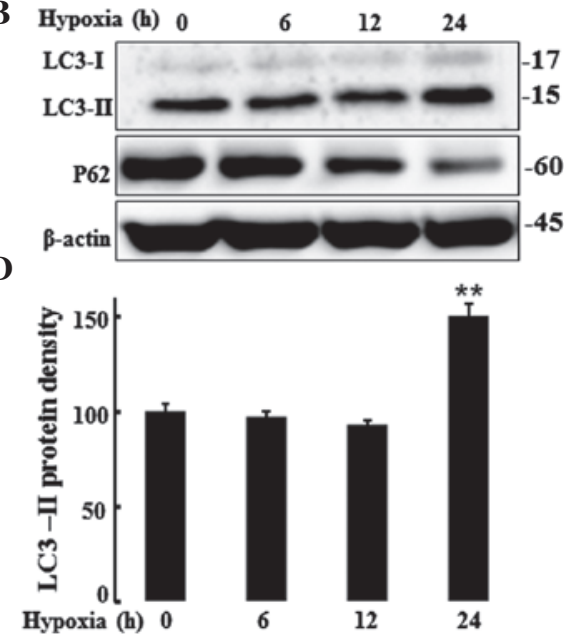

Figure 2. HIF-1 $\alpha$ induces the expression of autophagy markers in hypoxic HaCaT cells. (A and B) The cells were pre-incubated under hypoxic conditions (1\% $\mathrm{O}_{2}$ for $0,6,12$ or 24 h), followed by western blot analysis of HIF-1 $\alpha$, LC3 and P62. $\beta$-actin was used as a loading control. (C and D) Quantification of P62 and LC3-II levels. Values are presented as the mean \pm standard error of three experiments. ${ }^{*} \mathrm{P}<0.05,{ }^{* *} \mathrm{P}<0.001 \mathrm{vs}$. control (0 h hypoxia). LC 3 , light chain 3 ; HIF-1 $\alpha$, hypoxia-inducible factor $1 \alpha$.

blot analysis (Fig. 2B-D). Decreased p62 protein levels were observed under hypoxic conditions as compared to those under normoxic conditions. By contrast, the autophagic flux marker, LC3-II was increased after $24 \mathrm{~h}$ of hypoxia. These results indicated that hypoxia increased autophagic flux.

Autophagic flux inhibitors induce apoptosis in hypoxic keratinocytes. Autophagy inhibitors were utilized to inhibit autophagic flux in order to examine the protective role of autophagy in TRAIL-induced cell death. Hypoxia prevented TRAIL-induced apoptotic death in cells not treated with autophagy inhibitors. By contrast, treatment with the autophagy inhibitors 3-MA and CQ blocked hypoxic inhibition of TRAIL-induced apoptosis (Fig. 3A). Cell viability and LDH assays confirmed that hypoxia-mediated induction of autophagy protected hypoxic HaCaT cells from TRAIL-induced apoptosis (Fig. 3B and C). These results demonstrate that TRAIL-induced apoptosis was blocked by autophagy inhibitors.

Autophagic flux is blocked by autophagy inhibitors. To confirm the inhibition of autophagic flux by the autophagy inhibitors, expression of the autophagy marker, LC3 and the levels of p62 protein were assessed by western blot analysis. Treatment with 3-MA decreased the levels of LC3 in hypoxic HaCaT cells, confirming the inhibition of autophagy; however, treatment 

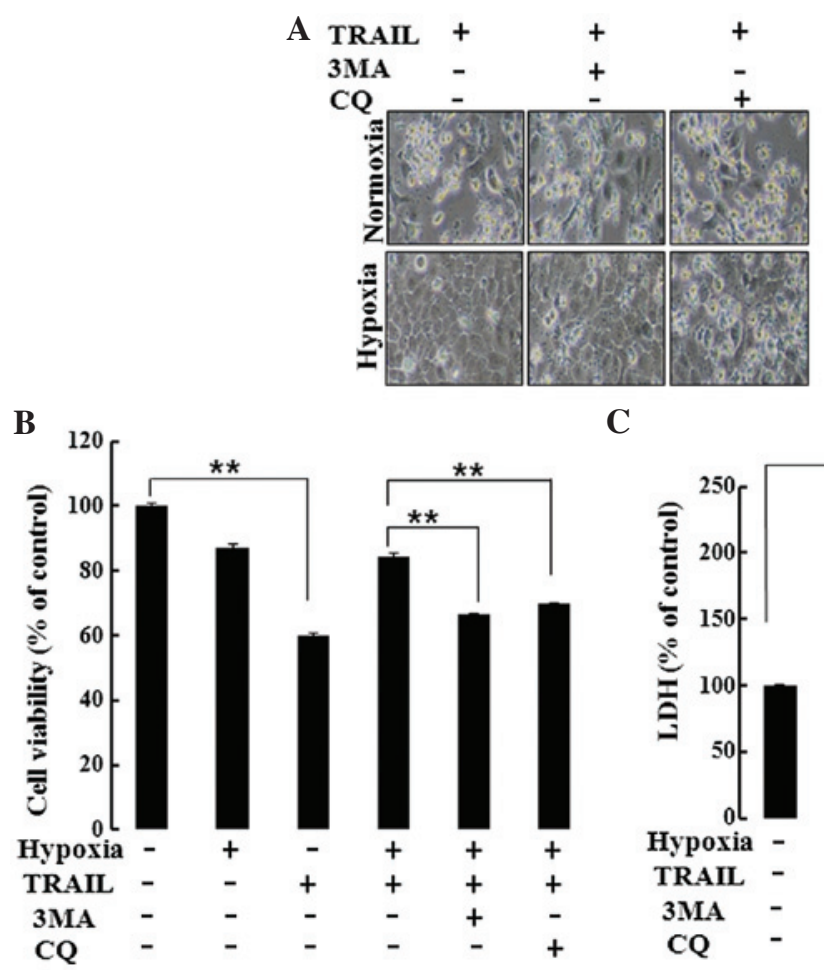

C

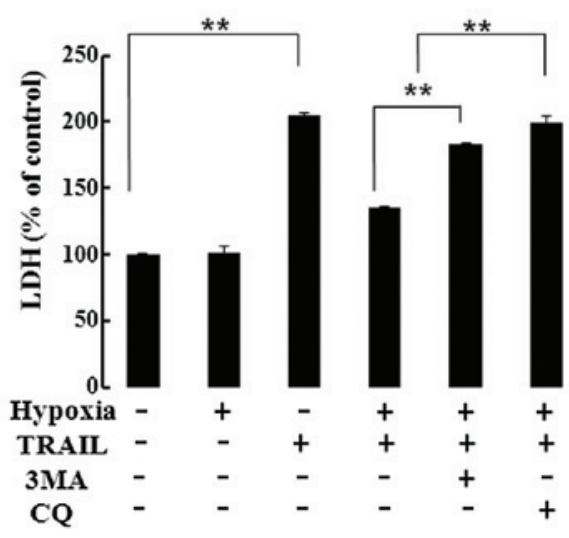

Figure 3. Autophagic flux inhibitors induce apoptosis in hypoxic keratinocytes. (A) Cell morphology was observed by light microscopy (magnification, x200). HaCaT cells were exposed to $200 \mathrm{ng} / \mathrm{ml}$ TRAIL for $6 \mathrm{~h}$ under normoxia or hypoxia $\left(1 \% \mathrm{O}_{2}\right.$ for $\left.24 \mathrm{~h}\right)$. (B) The viability of the treated cells was measured by crystal violet staining. HaCaT cells were pre-treated with autophagy inhibitors ( $200 \mu \mathrm{M} 3-\mathrm{MA}$ or $50 \mathrm{nMCQ})$ for $3 \mathrm{~h}$ and then exposed to TRAIL (200 ng) for $6 \mathrm{~h}$ under hypoxic conditions $\left(1 \% \mathrm{O}_{2}\right.$ for $24 \mathrm{~h}$ ). (C) The cells were treated with $200 \mathrm{ng} / \mathrm{ml}$ TRAIL for $6 \mathrm{~h}$ under normoxia after being treated with the autophagy inhibitors (3-MA or CQ; $3 \mathrm{~h}$ ) under hypoxia $\left(1 \% \mathrm{O}_{2}\right.$ for $\left.24 \mathrm{~h}\right)$, and $\mathrm{LDH}$ release into the cell culture medium was measured. Values are expressed as the mean \pm standard error $(\mathrm{n}=2) .{ }^{* *} \mathrm{P}<0.01$. MA, methyladenine; $\mathrm{CQ}$, chloroquine; $\mathrm{LDH}$, lactate dehydrogenase; TRAIL, tumor necrosis factor-related apoptosis-inducing ligand.

A

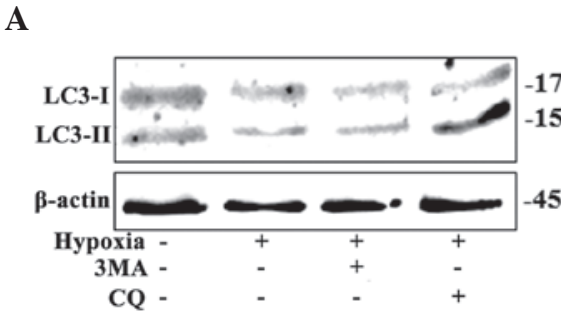

B

$\mathbf{C}$
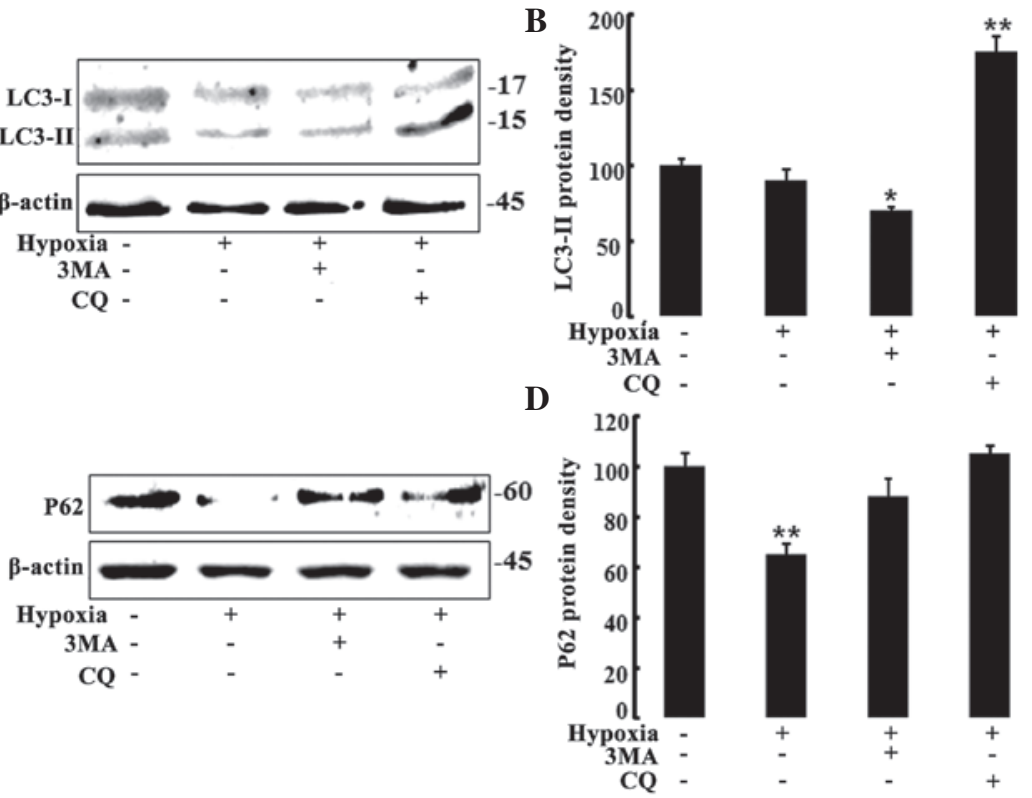

Figure 4. Confirmation of inhibited autophagic flux by the autophagy inhibitors. The cells were pre-treated with the autophagy inhibitors (200 $\mu \mathrm{M} 3-\mathrm{MA}$ or $50 \mathrm{nM} \mathrm{CQ}$ ) for $1 \mathrm{~h}$ under hypoxic conditions $\left(1 \% \mathrm{O}_{2}\right.$ for $24 \mathrm{~h}$ ), followed by western blot analysis of (A and B) LC3 and (C and D) P62 proteins in HaCaT cells. $\beta$-actin was used as a loading control. Values are presented as the mean \pm standard error of three experiments. ${ }^{*} \mathrm{P}<0.05,{ }^{* * *} \mathrm{P}<0.001 \mathrm{vs}$. control. LC3, light chain 3; MA, methyladenine; CQ, chloroquine.

with CQ increased the levels of LC3-II (Fig. 4A and B). Autophagy inhibitor-treated cells demonstrated increased p62 protein levels (Fig. 4C and D). As 3-MA inhibits conversion of
LC3-I to LC3-II and CQ blocks fusion of autophagolysosomes, treatment with 3-MA decreased the LC3-II levels whereas CQ increased the LC3-II levels. These results confirmed that 
autophagic flux was inhibited by the autophagy inhibitors, 3-MA and CQ.

\section{Discussion}

The main goal of the present study was to demonstrate the role autophagy has in the inhibition of TRAIL-induced apoptosis by hypoxia. The results suggested a therapeutic potential for autophagic flux inhibitors in chemotherapeutic intervention strategies that involve the use of TRAIL for skin cancer.

TRAIL is a member of the TNF superfamily that induces cancer cell death and normal human epidermal keratinocyte death by death receptors, including DR4 and DR5 (2). Death receptors mediate TRAIL-induced apoptosis, whereas the decoy receptors inhibit apoptosis induced by TRAIL (1). TRAIL induces cornification in normal human keratinocytes, which is a specific form of programmed cell death (19). Early studies discovered that TRAIL induces tissue injury (including apoptosis and inflammation) in human endothelial cells and may influence tumor therapies using TRAIL $(2,3,20)$. However, the roles of TRAIL treatment in human epidermal keratinocytes are not well understood. The results of the present study showed that TRAIL-treated HaCaT cells had a $30-60 \%$ reduced viability under normoxia, whereas cell viability was not markedly affected by TRAIL under hypoxic conditions (5\% reduction).

Changes in oxygen levels are tightly linked to metabolism, apoptosis, the cell cycle and cell signaling. HIF-1 is a transcriptional factor with $\alpha$ - and $\beta$-sub-units that mediates changes in gene expression under hypoxic conditions and is essential for skin development and wound healing $(7,8)$. Furthermore, a recent study indicated that p62 protein expression, an autophagic flux marker, decreases under hypoxic conditions (12). The present study showed that p62 expression decreased and LC3 expression increased under hypoxic conditions. These results suggested that the expression of HIF-1 induced autophagic flux in human epidermal keratinocytes and inhibited TRAIL-induced apoptosis.

Furthermore, the autophagy inhibitors 3-MA and CQ were used under hypoxic conditions to confirm the inhibition of cell death by autophagic flux. HaCaT cells showed a reduced resistance to the apoptotic effect of TRAIL during treatment with the autophagic inhibitors under hypoxic conditions. These results indicated that autophagic flux suppressed TRAIL-induced apoptosis.

TRAIL treatmenthas been shown to induceautophagy-dependent cell death in a variety of cancer cells $(21,22)$. The $\mathrm{p} 62$ protein, a marker of autophagy, is important in the degradation of polyubiquitinated proteins via the autophagy pathway (23). Recently, high autophagic flux with clearance of the p62 protein was detected in TRAIL-resistant cells; however, TRAIL-sensitive cells exhibited low autophagic flux and accumulation of the p62 protein (24). Data from the present study demonstrated that hypoxia inhibits TRAIL-induced cell death (via observation of the reduction of p62 protein), however treatment with TRAIL plus an autophagy inhibitor increased TRAIL-induced apoptosis, which was demonstrated by an accumulation of the p62 protein (Figs. 3A and 4D).

In conclusion, the results of the present study suggested that the autophagic flux induced by hypoxia inhibits
TRAIL-induced apoptosis. To the best of our knowledge, the present study was the first to identify that TRAIL-induced apoptosis was inhibited by hypoxia in keratinocytes, but that autophagy inhibitors were able to restore the susceptibility of keratinocytes to TRAIL-induced apoptosis under hypoxic conditions. These findings provided insight into the molecular mechanisms of keratinocyte apoptosis and the beneficial effects of TRAIL in skin cancer therapy. It is recommended that autophagy inhibitors are used to enhance the efficiency of TRAIL in the treatment of skin cancer.

\section{Acknowledgements}

The present study was supported by the National Research Foundation of the Korea Grant funded by the Korean Government (no. 2013R1A2A2A01009614).

\section{References}

1. Johnstone RW, Frew AJ and Smyth MJ: The TRAIL apoptotic pathway in cancer onset, progression and therapy. Nat Rev Cancer 8: 782-798, 2008.

2. Wu NL, Lee TA, Tsai TL and Lin WW: TRAIL-induced keratinocyte differentiation requires caspase activation and p63 expression. J Invest Dermatol 131: 874-883, 2011.

3. Li JH, Kirkiles-Smith NC, McNiff JM and Pober JS: TRAIL induces apoptosis and inflammatory gene expression in human endothelial cells. J Immunol 171: 1526-1533, 2003.

4. Semenza GL: Hypoxia-inducible factor 1: control of oxygen homeostasis in health and disease. Pediatr Res 49: 614-617, 2001.

5. Hu Y, Kirito K, Yoshida K, Mitsumori T, Nakajima K, Nozaki Y, Hamanaka S, Nagashima T, Kunitama M, Sakoe K and Komatsu N: Inhibition of hypoxia-inducible factor-1 function enhances the sensitivity of multiple myeloma cells to melphalan. Mol Cancer Ther 8: 2329-2338, 2009.

6. Kaelin WG Jr: Treatment of kidney cancer: insights provided by the VHL tumor-suppressor protein. Cancer 115 (Suppl 10): S2262-S2272, 2009.

7. Tandara AA and Mustoe TA: Oxygen in wound healing-more than a nutrient. World J Surg 28: 294-300, 2004.

8. LaVan FB and Hunt TK: Oxygen and wound healing. Clin Plast Surg 17: 463-472, 1990.

9. Evans NT and Naylor PF: The systemic oxygen supply to the surface of human skin. Respir Physiol 3: 21-37, 1967.

10. Stewart FA, Denekamp J and Randhawa VS: Skin sensitization by misonidazole: a demonstration of uniform mild hypoxia. Br J Cancer 45: 869-877, 1982.

11. Stucker M, Struk A, Altmeyer P, Herde M, Baumgärtl H and Lübbers DW: The cutaneous uptake of atmospheric oxygen contributes significantly to the oxygen supply of human dermis and epidermis. J Physiol 538: 985-994, 2002.

12. Pursiheimo JP, Rantanen K, Heikkinen PT, Johansen T and Jaakkola PM: Hypoxia-activated autophagy accelerates degradation of SQSTM1/p62. Oncogene 28: 334-344, 2009.

13. Levine B and Kroemer G: Autophagy in the pathogenesis of disease. Cell 132: 27-42, 2008.

14. Delgado MA, Elmaoued RA, Davis AS, Kyei G and Deretic V: Toll-like receptors control autophagy. EMBO J 27: 1110-1121, 2008.

15. Hussey S, Travassos LH and Jones NL: Autophagy as an emerging dimension to adaptive and innate immunity. Semin Immunol 21: 233-241, 2009.

16. Zhao Y, Zhang CF, Rossiter H, Eckhart L, König U, Karner S, Mildner M, Bochkov VN, Tschachler E and Gruber F: Autophagy is induced by UVA and promotes removal of oxidized phospholipids and protein aggregates in epidermal keratinocytes. J Invest Dermatol 133: 1629-1637, 2013

17. Lee HM, Shin DM, Yuk JM, Shi G, Choi DK, Lee SH, Huang SM, Kim JM, Kim CD, Lee JH and Jo EK: Autophagy negatively regulates keratinocyte inflammatory responses via scaffolding protein p62/SQSTM1. J Immunol 186: 1248-1258, 2011.

18. Seo JS, Seol JW, Moon MH, Jeong JK, Lee YJ and Park SY: Hypoxia protects neuronal cells from human prion protein fragment-induced apoptosis. J Neurochem 112: 715-722, 2010. 
19. Candi E, Rufini A, Terrinoni A,Dinsdale D, Ranalli M,Paradisi A, De Laurenzi V, Spagnoli LG, Catani MV, Ramadan S, et al: Differential roles of p63 isoforms in epidermal development: selective genetic complementation in p63 null mice. Cell Death Differ 13: 1037-1047, 2006.

20. Eberle J, Fecker LF, Forschner T, Ulrich C, Röwert-Huber J and Stockfleth E: Apoptosis pathways as promising targets for skin cancer therapy. Br J Dermatol 156 (Suppl 3): 18-24, 2007.

21. Mora R, Abschuetz A, Kees T, Dokic I, Joschko N, Kleber S Geibig R, Mosconi E, Zentgraf H, Martin-Villalba A and Régnier-Vigouroux A: TNF-alpha- and TRAIL-resistant glioma cells undergo autophagy-dependent cell death induced by activated microglia. Glia 57: 561-581, 2009.
22. Herrero-Martín G, Høyer-Hansen M, García-García C, Fumarola C, Farkas T, López-Rivas A and Jäättelä M: TAK1 activates AMPK-dependent cytoprotective autophagy in TRAIL-treated epithelial cells. EMBO J 28: 677-685, 2009.

23. Itakura E and Mizushima N: p62 Targeting to the autophagosome formation site requires self-oligomerization but not LC3 binding. J Cell Biol 192: 17-27, 2011.

24. Singh K, Sharma A, Mir MC, Drazba JA, Heston WD Magi-Galluzzi C, Hansel D, Rubin BP, Klein EA and Almasan A: Autophagic flux determines cell death and survival in response to Apo2L/TRAIL (dulanermin). Mol Cancer 13: 70 , 2014. 